Rev Biomed 2002; 13:73-75.

\title{
Informe del fenómeno de marea roja en Bahía de Banderas Jalisco- Nayarit, octubre- noviembre de 2001.
}

Carta al Editor

María del Carmen Cortés-Lara.

Departamento de Ciencias, Centro Universitario de la Costa, Universidad de Guadalajara. Puerto Vallarta, Jalisco, México.

Aproximadamente el día 4 de octubre del año en curso se presentó en las aguas costeras de la Bahía de Banderas Jalisco, un nuevo evento continuo de marea roja. Las primeras manifestaciones del florecimiento algal se observaron hacia la parte norte de la bahía afectando fuertemente las áreas de Nuevo Vallarta, Bucerías, La Cruz de Huanacaxtle, Destiladeras, Punta de Mita, hasta la zona de Platanitos, Nayarit.

Los parches de este dinoflagelado se han desplazado rápidamente en toda la bahía a manera de discoloraciones irregulares rojo-óxido muy intenso, acompañado de un fuerte mal olor debido a la descomposición de la materia orgánica.

El análisis de las muestras de agua revelaron nuevamente la presencia del dinoflagelado ictiotóxico Cochlodinium catenatum como la especie causal de la marea roja (figuras 1 y 2). Las densidades se han registrado desde 3 a 7 células por mililitro en Los Arcos y Hotel Sheraton de Puerto Vallarta al sur de la bahía.

En cambio, se reportan concentraciones mayores del orden de 3,008 a 1,192 células por mililitro $(3,008,000$ a 1,192,000 millones de células por litro) en las localidades de la dársena portuaria de Puerto Vallarta, el área turística de Nuevo Vallarta, Destiladeras, Bucerías y La Cruz de Huanacaxtle al norte de Bahía de Banderas.

Esta especie se ha descrito como un organismo cosmopolita de aguas templadas y tropicales, a manera de cadenas gyrodinoides con células compactas (de 4 a 6 , hasta muy raramente 16) cuyos individuos son de talla pequeña elipsoidales, epiteca cónica, hipoteca bilobada cuando las células son libres, con un cíngulo excavado y desplazado.

Solicitud de sobretiros: M.C. María del C. Cortés-Lara. Depto. de Ciencias, Centro Univeristario de la Costa, Universidad de Guadalajara,Puerto Vallarta, Jalisco, México.Tel.: 226-22-15. E-mail: carmenc@pv1.udg.mx. 


\section{M del C Cortés-Lara.}

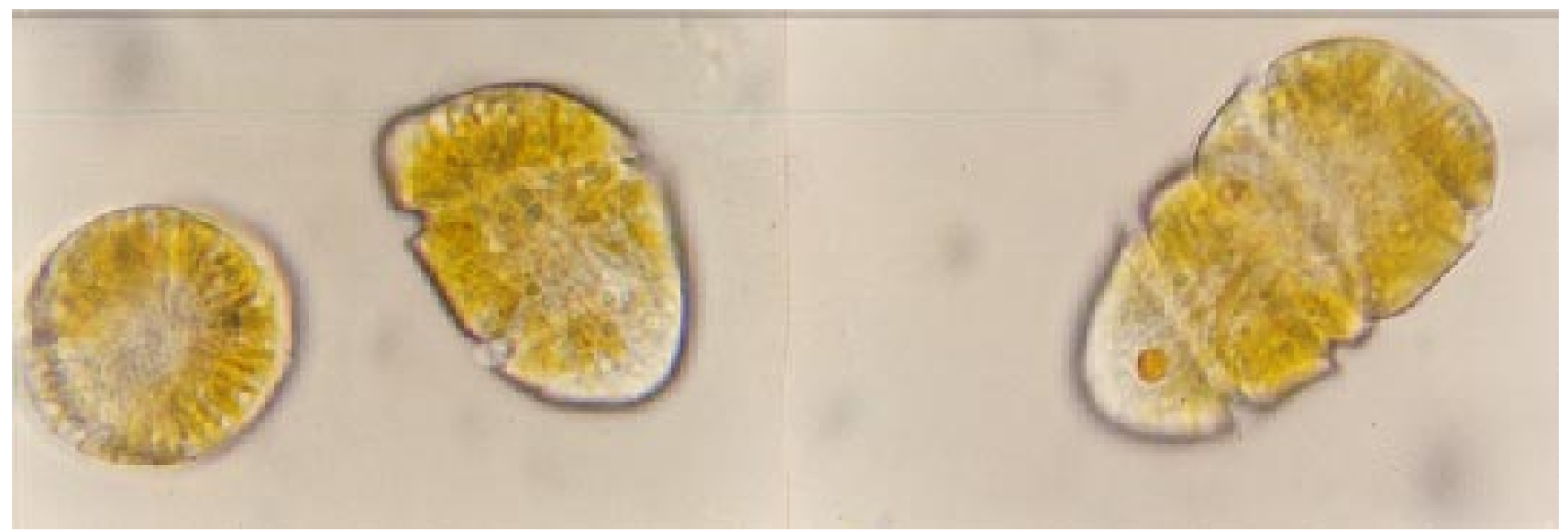

Figuras 1 y 2.- Microorganismo causante de la marea roja (Cochlodinium catenatum).

Existen reportes previos de la ocurrencia de esta microalga en Bahía de Banderas durante el verano del 2000. El evento tuvo una duración de 18 semanas en donde se afectaron al menos 23 kilómetros de costa particularmente en áreas en donde la actividad turística tiene gran afluencia, como es el caso de Puerto Vallarta y Nuevo Vallarta.

En el verano del 2000 el fenómeno se definió como el más extenso y de mayor duración de la que se tiene conocimiento en esta localidad y en las costas de México, despertando alarma en la población como resultado de la muerte de peces marinos.

Su presencia en el Pacífico Mexicano se ha mencionado también en la Bahía de Manzanillo Colima, en Bahía de Mazatlán y en la Ensenada de La Paz Baja California.

A pesar de que las muestras de Cochlodinium cateantum no han confirmado la presencia de ficotoxinas PSP y DSP (1), se sugiere que Cochlodinium spp. podrían secretar sustancias neurotóxicas, hemolíticas y hematoglutinantes (2).

Por otro lado es importante considerar que Cochlodinium spp. son caracterizados como organismos ROS generadores de radicales $\mathrm{O}_{2}$ tales como aniones de superóxicos $\left(\mathrm{O}_{2}^{-}\right)$ hidroxílos $(\mathrm{OH})$ y peróxidos de hidrógeno $\left(\mathrm{H}_{2} \mathrm{O}_{2}\right)$ para explicar la mortandad de peces en la bahía (3). Tales compuestos causan daños oxidativos a nivel de funcionamiento y estructura de las células de las agallas de los peces, reduciendo la capacidad para la transferencia de oxígeno.

En la mayoría de los casos de muerte masiva de peces, la calidad de la carne se deteriora rápidamente por lo que no se recomienda la venta ni el consumo debido al alto grado de descomposición de los peces.

Es importante mencionar que este evento de marea roja puede llegar a afectar la salud humana al entrar en contacto directo con aguas de zonas de pesca o lugares de recreación, dando como resultado irritación en la piel, mucosa nasal, ocular o dermatitis en general. En todos estos casos éstas no pasan de ser molestias leves que no requieren tratamiento especializado.

Palabras clave: marea roja, Puerto Vallarta, Cochlodinium catenatum.

\section{REFERENCIAS.}

1.- Onoue, Y., Nozawa, K., Kumanda, K., Takeda, K.

\section{Revista Biomédica}


Marea Roja en Bahía de Banderas, México.

And Aramaki, T. Toxicity of Cochlodinium polykrikoides type '78 Yatsushiro occurring in Yatsushiro Sea Bull Jpn Soc Sci Fish 1985; 51: 147.

2.- Onoue, Y. and Nozawa, K. $\left(1989^{\mathrm{a}}\right)$. Separation of toxins from harmful red tides occurring along the coast of Kagoshima prefecture. In. Okaichi. T., Anderson, D. M. And Nemoto, T. (eds). Red Tides: Biology, Environmental Sience and Toxicology. Elsevier, New York, pp. 371-374.

3.- Chang-Sook Kim, Sam Geun Lee, Chang Kyu Lee, Hak Gyoon Kim and Jin Jung. (1999). Reactive Oxygen species as causative agents in the icthyotoxicity of red tide dinoflagellate Cochlodinium polykrikoides. Journal of Plankton Research. Vol. 21. No. 11 pp. 2105-2115.

\section{Fe de Erratas:}

El nombre del primer autor del articulo "Chromosomal aberrations in smokers exposed to metallic dust in mint factory" del volumen 11, No. 2 páginas 87-90, de Abril-Junio de 2000 es Padmaja Reddy en vez de Padmala Reddy.

\section{Correction:}

The name of the first author of the article "Chromosomal aberrations in smokers exposed to metallic dust in mint factory", volume 11, number 2, pages 87-90, April-June 2000, is Padmaja Reddy instead of Padmala Reddy. 